\title{
On Elliptic Equations and Systems with Critical Growth in Dimension Two
}

\author{
Bernhard Ruf \\ Dipartimento di Matematica, Università degli Studi \\ Via Saldini 50, 20133 Milano, Italy, ruf@mat.unimi.it \\ Dedicated to Professor S. Nikolskii on his 100th birthday
}

\begin{abstract}
In this paper we consider nonlinear elliptic equations of the form

$$
\left\{\begin{aligned}
-\Delta u & =g(u), & & \text { in } \quad \Omega, \\
u & =0 & , & \text { on } \partial \Omega,
\end{aligned}\right.
$$

and Hamiltonian type systems of the form :

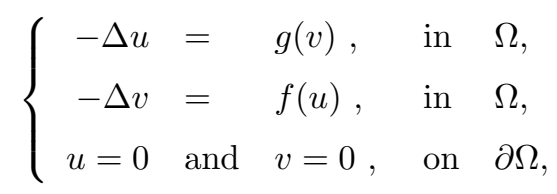

where $\Omega$ is a bounded domain in $\mathbb{R}^{2}$, and $f, g \in C(\mathbb{R})$ are superlinear nonlinearities. In two dimensions the maximal growth (= critical growth) of $f, g$ (such that the problem can be treated variationally) is of exponential type, given by Pohozaev-Trudinger type inequalities. We discuss existence and nonexistence results related to critical growth for the equation and the system.

The natural framework for such equations and systems are Sobolev spaces, which give in most cases an adequate answer concerning the maximal growth involved. However, we will see that for the system in dimension 2, the Sobolev embeddings are not sufficiently fine to capture the true maximal growths. We will show that working in Lorentz spaces gives better results.
\end{abstract}




\section{Introduction}

In this paper we consider nonlinear elliptic equations of the form

$$
\left\{\begin{aligned}
-\Delta u & =g(u), & & \text { in } \quad \Omega, \\
u & =0 & , & \text { on } \partial \Omega,
\end{aligned}\right.
$$

and Hamiltonian type systems of the form :

$$
\left\{\begin{aligned}
-\Delta u=g(v), & \text { in } \Omega, \\
-\Delta v=f(u), & \text { in } \Omega, \\
u=0 \text { and } v=0, & \text { on } \partial \Omega,
\end{aligned}\right.
$$

where $\Omega$ is a bounded domain in $\mathbb{R}^{2}$. Here, $f$ and $g$ are continuous and superlinear functions.

Consider first the equation (E): solutions correspond to critical points of the energy functional

$$
I(u)=\frac{1}{2} \int_{\Omega}|\nabla u|^{2} d x-\int_{\Omega} G(u) d x,
$$

where $G(t)=\int_{0}^{t} g(s) d s$ denotes the primitive of $g(t)$. Considering this functional on the Sobolev space $H_{0}^{1}(\Omega)$, one obtains by the well-known inequality of Pohozaev [21] and Trudinger [26] a natural growth restriction (critical growth) on $G(t)$. This inequality says that if $u$ is a $H_{0}^{1}(\Omega)$ function, then the integral $\int_{\Omega} e^{u^{2}} d x$ is finite. Furthermore, by the sharpened form of J. Moser [20] one has

$$
\sup _{\|u\|_{H_{0}^{1}} \leq 1} \int_{\Omega} e^{\alpha u^{2}} d x \quad \begin{cases}<+\infty, & \text { if } \alpha \leq 4 \pi \\ =+\infty, & \text { if } \alpha>4 \pi\end{cases}
$$

The problems which arise naturally are:

1.) existence of solutions if $g$ has subcritical growth

2.) non-compactness and related phenomena if $g$ has critical growth

Turning to the system (S), we first note that the natural functional associated to (S) has the form

$$
J(u, v)=\int_{\Omega} \nabla u \nabla v d x-\int_{\Omega} F(u) d x-\int_{\Omega} G(v) d x,
$$

where $F$ and $G$ are again the primitives of $f$ and $g$, respectively. The functional $J(u, v)$ can again be defined on the space $H_{0}^{1}(\Omega) \times H_{0}^{1}(\Omega)$, and then one finds as before a maximal growth of type

$$
f(t) \sim e^{t^{2}} \quad, \quad g(t) \sim e^{t^{2}}
$$

Again, the same questions 1.) and 2.) as for the equation $(E)$ arise. We note that the functional $J(u, v)$ is considerably more difficult to handle than $I(u)$, since $J(u, v)$ is 
strongly indefinite (i.e. positive and negative definite on $\infty$-dimensional subspaces, respectively). On the other hand, we note that the choice of the space $H_{0}^{1} \times H_{0}^{1}$ is not as compelling as in the case of $I(u)$. Indeed, the term $\int_{\Omega} \nabla u \nabla v$ in $J(u, v)$ is also defined on $W_{0}^{1, \alpha}(\Omega) \times W_{0}^{1, \beta}(\Omega), \frac{1}{\alpha}+\frac{1}{\beta}=1$, by estimating

$$
\left|\int_{\Omega} \nabla u \nabla v d x\right| \leq\|\nabla u\|_{\alpha}\|\nabla v\|_{\beta}
$$

We note that the choice $0<\alpha<2$ implies by Sobolev's embedding theorem

$$
W_{0}^{1, \alpha}(\Omega) \subset L^{\frac{N \alpha}{N-\alpha}}(\Omega)
$$

and furthermore, since $\beta=\frac{\alpha}{\alpha-1}>2$, we get

$$
W_{0}^{1, \beta}(\Omega) \subset L^{\infty}(\Omega)
$$

Thus, we have a maximal growth of polynomial type for $F$ :

$$
|F(s)| \leq|s|^{\frac{N \alpha}{N-\alpha}}
$$

and no growth restriction on the nonlinearity $G$. So this choice of spaces brings us immediatly outside of the range of exponential nonlinearities.

However, a refined choice of spaces is possible, namely the so-called Lorentz spaces, or more precisely Sobolev-Lorentz spaces, which are interpolation space between the usual Sobolev spaces. This approach allows to derive an "exponential critical curve", in complete analogy to the so-called "critical hyperbola" in the case of $N \geq 3$. Again, the same questions 1.) and 2.) may now be asked with respect to this new critical growth.

\section{The equation (E)}

In this section we consider equation (E). We say that $g(t)$ has subcritical growth if

$$
\lim _{|t| \rightarrow \infty} \frac{g(t)}{e^{\alpha t^{2}}}=0, \text { for every } \alpha>0
$$

and $g(t)$ has critical growth if there exists $\alpha_{0}>0$ such that

$$
\lim _{|t| \rightarrow \infty} \frac{g(t)}{e^{\alpha t^{2}}}=0 \quad \text { if } \alpha>\alpha_{0} \quad, \quad \text { and } \quad \lim _{|t| \rightarrow \infty} \frac{g(t)}{e^{\alpha t^{2}}}=+\infty \quad \text { if } 0<\alpha<\alpha_{0} .
$$

We first consider

\subsection{Subcritical growth}

Concerning subcritical growth, one has the following existence result: 
Theorem 2.1 (see [4], [14])

Assume that $f: \bar{\Omega} \times \mathbb{R} \rightarrow \mathbb{R}$ is continuous, and satisfies

(H1) there exist constants $t_{0}>0$ and $M>0$ such that

$$
\begin{aligned}
& 0<F(x, t)=\int_{0}^{t} f(x, s) d s \leq M|f(x, t)|, \forall|t| \geq t_{0}, \forall x \in \Omega \\
& 0<F(x, t) \leq \frac{1}{2} f(x, t) t, \forall t \in \mathbb{R} \backslash\{0\}, \forall x \in \Omega
\end{aligned}
$$

Then problem $(E)$ has a nontrivial solution. Moreover, if $f(x, t)$ is an odd function in $t$, then equation $(E)$ has infinitely many solutions.

Proof. The proof follows (by now) standard lines: the assumptions guarantee that the functional has a mountain pass structure around the origin, cf. [3],[23]. The subcritical growth yields compactness (through the Pohozaev-Trudinger inequality, see also P.L. Lions [19]), and hence the critical level is attained.

\subsection{Critical growth}

We consider now equation (E) with critical growth in the sense specified above. For reference, let us first recall briefly the situation in $N \geq 3$, which has been studied extensively, and which is by now very well understood. Here, critical growth is given by $g(s)=|s|^{2^{*}-2} s$ where $2^{*}=\frac{2 N}{N-2}$ is the limiting Sobolev exponent, and so the corresponding equation is

$$
-\Delta u=|u|^{2^{*}-2} u
$$

One knows that:

- equation (4) has, on all of $\mathbb{R}^{N}$, the family of solutions $u_{\lambda}(x)=c\left(\frac{\lambda}{\lambda^{2}+\left|x-x_{0}\right|^{2}}\right)^{\frac{N-2}{2}}$, which "concentrate" as $\lambda \rightarrow 0$ (see G. Talenti [25])

- equation (4) has no nontrivial solutions on bounded and starshaped domains: this is due to the seminal Pohozaev identitiy [22]

- the non-existence may also be understood as a result of the loss of compactness due the concentrating family of solutions on $\mathbb{R}^{N}$

- existence of solutions can be recovered if (4) is perturbed by suitable lower order terms. This is the ground-breaking result by Brezis-Nirenberg [6]. The argument goes as follows: due to the concentrating sequence, the noncompactness of the perturbed functional occurs at a specific (and explicitly known) level. The (suitable) perturbations of the functional produce a minimax level below the non-compactness level; thus, there is compactness at this level, and hence one may prove the existence of a critical point at this level

For the case $N=2$ the situation is more complicated, and the known results are less complete. The difficulties start already with the fact that there is no natural "model problem" for the critical case. Thus, let us write the "critical" equation (with $\alpha_{0}=4 \pi$, see (3)) in the form

$$
-\Delta u=h(u) e^{4 \pi u^{2}}
$$


where $h \in C(\mathbb{R})$ is subcritical, i.e. satisfying condition (2). The question is now whether there exist optimal (= sharp) conditions on $h(t)$ such that we have again the situation of non-existence and existence of solutions.

Related to the study of this question is the behavior of the supremum in (1). Indeed, it came as a surprise when L. Carleson - A. Chang [8] proved in 1986 that the supremum in (1) is attained on the unit ball in $\mathbb{R}^{2}$ (this result was extended to arbitrary domains in $\mathbb{R}^{2}$ by M. Flucher [16]). Carleson and Chang prove their result by the following steps:

- the supremum is radial, and thus characterized by an ODE (the radial equation)

- if the supremum is not attained, then there exists a maximizing sequence which tends weakly to 0 and concentrates in the origin

- determine an explicit upper bound, namely $\int_{B_{1}(0)} e^{4 \pi u_{n}^{2}} \leq(1+e) \pi$, for any normalized concentrating sequence $\left(u_{n}\right) \in H_{0}^{1}\left(B_{1}\right)$

- provide an explicit normalized function $w \in H_{0}^{1}\left(B_{1}\right)$ with $\int_{B_{1}} e^{4 \pi w^{2}}>(1+e) \pi$

Clearly, $(1+e) \pi$ takes the rôle of the (highest) non-compactness level, analogous to the situation in $\mathbb{R} \geq 3$ described above, and since the supremum lies above this non-compactness level, it is attained.

In a recent paper by De Figueiredo, do Ó and the author [15] the following explicit normalized concentrating and maximizing sequence for $(1+e) \pi$ was constructed:

For $n \in \mathbb{N}$ set $\delta_{n}=\frac{2 \log n}{n}$ and $A_{n}=\frac{1}{e n^{2}}+O\left(\frac{1}{n^{4}}\right)$; then define

$$
y_{n}(|x|)= \begin{cases}\left(\frac{1-\delta_{n}}{n}\right)^{1 / 2} \log \frac{1}{|x|} & 1 / n \leq|x| \leq 1 \\ \frac{1}{\left(n\left(1-\delta_{n}\right)\right)^{1 / 2}} \log \frac{A_{n}+1}{A_{n}+n|x|}+\left(n\left(1-\delta_{n}\right)\right)^{1 / 2}, & 0 \leq|x| \leq 1 / n\end{cases}
$$

This sequence allows to give a new proof of the last step in the argument of CarlesonChang, and also a slight generalization of their result. In view of this and the above remarks, it is of interest to consider

$$
\sup _{u \in H_{0}^{1}(\Omega),\|u\|=1} \int_{\Omega} h(u) e^{4 \pi u^{2}}=S
$$

and give optimal (= sharp) conditions on the subcritical function $h(t)$ such that the supremum $S$ is attained, respectively not attained.

\subsection{Critical growth: existence}

For the corresponding equation

$$
\left\{\begin{aligned}
-\Delta u & =h(u) e^{4 \pi u^{2}}, \text { in } \Omega \\
u & =0, \text { on } \partial \Omega
\end{aligned}\right.
$$

some progress has been made recently concerning the determination of an optimal subcritical function $h(t)$. We remark that concerning non-existence results, a 
fundamental difference to the case $N \geq 3$ is that (up to now) no suitable identity of Pohozaev type is known for the case $N=2$.

In [14] the following theorem was proved by de Figueiredo, Miyagaki and the author (see also Adimurthi [2]):

Theorem 2.2 Assume that $h \in C(\mathbb{R})$ and let $f(s)=h(s) e^{4 \pi s^{2}}$. Assume that

H1) $f(0)=0$

$H 2)$ there exist constants $s_{0}>0$ and $M>0$ such that

$$
0<F(s)=\int_{0}^{s} f(r) d r \leq M|f(s)|, \quad \forall|s| \geq s_{0}
$$

H3) $0<F(s) \leq \frac{1}{2} f(s) s, \forall s \in \mathbb{R} \backslash\{0\}$

Furthermore, let $d$ denote the inner radius of $\Omega$, i.e.

$$
d:=\text { radius of the largest ball } \subset \Omega
$$

Then equation (6) has a nontrivial solution provided that

$$
\lim _{|s| \rightarrow \infty} h(s) s=\beta>\frac{1}{2 \pi d^{2}}
$$

The proof of this theorem follows closely the scheme by Brezis-Nirenberg mentioned above, that is

- determine (explicitly) the level of non compactness

- use an explicit concentrating sequence and the hypothesis on $h(t)$ to show that the min-max level is below this non-compactness level

- thus, compactness is recovered and the existence of a solution follows

The concentrating sequence used in the proof of this theorem is the so-called Moser sequence given by

$$
w_{n}=\frac{1}{\sqrt{2 \pi}} \begin{cases}\frac{1}{(\log n)^{1 / 2}} \log \frac{1}{|x|} & \frac{1}{n} \leq|x| \leq 1 \\ (\log n)^{1 / 2} & 0 \leq|x| \leq \frac{1}{n}\end{cases}
$$

We remark that this sequence is not an optimal concentrating sequence; in fact, one easily calculates that

$$
\lim _{n \rightarrow \infty} \int_{B_{1}} e^{4 \pi w_{n}^{2}}=2 \pi<(1+e) \pi
$$

We remark that the condition $H 4$ ) in Theorem 2.2 may be slightly improved to

$$
\beta>\frac{1}{e \pi d^{2}}
$$

by using the optimal maximizing sequence (5) mentioned above. 


\subsection{Critical growth: non-existence}

Concerning non-existence, only a partial result is known; in the following theorem, the non-existence of a positive radial solution on $\Omega=B_{1}(0)$ is proved under conditions comparable to those of Theorem 2.2.

Theorem 2.3 (de Figueiredo-Ruf [13])

Let $\Omega=B_{1}(0)$. Suppose that $h \in C^{2}(\mathbb{R})$, and that there exist constants $r_{1}>0$ and $\sigma>0$ such that for some constants $K>0, c>0$ :

1. $h(r)=\frac{K}{r} \quad$ for $r \geq r_{1}$

2. $0 \leq h(r) \leq c K r^{1+\sigma}$, for $0 \leq r \leq r_{1}$.

Then there exists a constant $K_{0}>0$ such that for $K<K_{0}$ the equation

$$
\left\{\begin{aligned}
-\Delta u & =h(u) e^{4 \pi u^{2}}, & & \text { in } B_{1}(0) \subset \mathbb{R}^{2} \\
u & =0, & & \text { on } \partial B_{1}(0)
\end{aligned}\right.
$$

has no non-trivial radial solution.

We remark that by Gidas-Ni-Nirenberg [17] any positive solution of equation (7) is radial, and hence Theorem 2.3 says that equation (7) has no positive solution.

The proof of Theorem 2.3 uses techniques of the theory of ordinary differential equations, in particular the shooting method. More precisely, considering only the radial solutions on $\Omega=B_{1}(0)$, one can reduce equation ( 7$)$ to the radial equation

$$
\left\{\begin{array}{l}
u_{r} r+\frac{1}{r} u_{r}+h(u) e^{4 \pi u^{2}}=0 \quad \text { in }(0,1) \\
u^{\prime}(0)=u(1)=0
\end{array}\right.
$$

Using the transformation $t=-2 \log \frac{r}{2}$ and setting $y(t)=u(r)$ we obtain

$$
\left\{\begin{array}{l}
-y^{\prime \prime}=h(y) e^{4 \pi y^{2}-t}, \text { for } t>2 \log 2 \\
y(2 \log 2)=0, y^{\prime}(+\infty)=0
\end{array}\right.
$$

That is, we have transformed equation (8), which has a singularity in 0 , to equation (9) on $(2 \log 2,+\infty)$, thus transporting the singularity in 0 to $+\infty$. The shooting method consists now in considering solutions $y(t)$ of $(9)$ with $y^{\prime}(+\infty)=\gamma$, i.e. one shoots horizonally from infinity and tries to land at the point $2 \log 2$. The estimates to achieve this are delicate and lengthy, and are a refinement of the work of AtkinsonPeletier [3].

We summarize: if we assume that the asymptotic condition in the existence theorem 2.2 is optimal (at least on the unit ball $B_{1}$ ), then the major open problem may be stated as follows:

Find a good model equation (i.e. properties of $h(u)$ ) under which one may prove: existence of a non-trivial solution for $\lim _{t \rightarrow \infty} h(t) t>\frac{1}{e \pi}$, and non-existence of a solution for $\lim _{t \rightarrow \infty} h(t) t<\frac{1}{e \pi}$.

As already mentioned, what seems to be missing is a kind of Pohozaev identity to obtain a sharp non-existence result. 


\section{The system (S)}

We now consider the system of equations

$$
\left\{\begin{aligned}
-\Delta u & =g(v), \text { in } \Omega \\
-\Delta v & =f(u), \text { in } \Omega \\
\left.u\right|_{\partial \Omega} & =\left.v\right|_{\partial \Omega}=0
\end{aligned}\right.
$$

where $\Omega \subset \mathbb{R}^{2}$ is a bounded domain, and the nonlinearities $f$ and $g$ are again continuous functions with exponential growth.

As mentioned in the introduction, the natural functional associated to system $(S)$ is

$$
J(u, v)=\int_{\Omega} \nabla u \nabla v-\int_{\Omega} F(u)-\int_{\Omega} G(v)
$$

Considering this functional on the product of Sobolev spaces $H_{0}^{1}(\Omega) \times H_{0}^{1}(\Omega)$ we obtain as maximal growth for both nonlinearities $F(t) \sim e^{t^{2}}, G(t) \sim e^{t^{2}}$.

We recall that in dimension $N \geq 3$ different maximal growths can be obtained by either working with fractional Sobolev spaces: $H^{s}(\Omega) \times H^{t}(\Omega)$ (here $H^{t}(\Omega)$ is the space of functions whose derivative of order $t \in \mathbb{R}^{+}$lies in $L^{2}(\Omega)$; these spaces can be obtained via Fourier series or interpolation, see Adams-Fournier [1]), or with Banach spaces of type: $W^{1, \alpha}(\Omega) \times W^{1, \beta}(\Omega)$, with $\frac{1}{\alpha}+\frac{1}{\beta}=1$. The first approach was used by Hulshof-van der Vorst [18] and de Figueiredo-Felmer [9], and the second by de Figueiredo-do Ó-Ruf [15]. In both cases one obtains the so-called critical hyperbola

$$
\frac{1}{p+1}+\frac{1}{q+1}=1-\frac{2}{N},
$$

that is the critical growth $F(t) \sim|t|^{2^{*}}$ with $2^{*}=\frac{2 N}{N-2}$ of the scalar equation is replaced by $F(t)=|t|^{p+1}$ and $G(t)=|t|^{q+1}$ with $p+1$ and $q+1$ satisfying (10).

As already mentioned in the introduction, in the case of systems in two dimensions Sobolev spaces do not seem suitable to extend the notion of criticality; we thus turn now to Lorentz spaces.

\subsection{Lorentz spaces}

We begin by recalling the definition of a Lorentz space: For $\phi: \Omega \rightarrow \mathbb{R}$ a measurable function, we denote by

$$
\mu_{\phi}(t)=|\{x \in \Omega: \phi(x)>t\}| \quad, t \geq 0
$$

its distribution function. The decreasing rearrangement $\phi^{*}(s)$ of $\phi$ is defined by

$$
\phi^{*}(s)=\sup \left\{t>0 ; \mu_{\phi}(t)>s\right\}, 0 \leq s \leq|\Omega| .
$$

The Lorentz space $L(p, q)$ is given as follows: $\phi \in L(p, q), 1<p<\infty, 1 \leq q<\infty$, if

$$
\|\phi\|_{p, q}=\left(\int_{0}^{\infty}\left[\phi^{*}(t) t^{1 / p}\right]^{q} \frac{d t}{t}\right)^{1 / q}<+\infty
$$

We recall the following properties of Lorentz spaces (see Adams-Fourier [1]): 
1. $L(p, p)=L^{p}, 1<p<+\infty$

2. The following inclusions hold for $1<q<p<r<\infty$ :

$$
L^{r} \subset L(p, 1) \subset L(p, q) \subset L(p, p)=L^{p} \subset L(p, r) \subset L^{q}
$$

3. Hölder inequality:

$$
\left|\int_{\Omega} f g d x\right| \leq\|f\|_{p, q}\|g\|_{p^{\prime}, q^{\prime}}, \text { where } p^{\prime}=\frac{p}{p-1}, q^{\prime}=\frac{q}{q-1}
$$

Furthermore, we recall the following embedding results:

Theorem A: Suppose that $1 \leq p<N$, and that $\nabla u \in L(p, q)$; then $u \in L\left(p^{*}, q\right)$, where $p^{*}=\frac{N p}{N-p}$ and $1 \leq q<\infty$.

For the next theorem, see H. Brezis [5]:

Theorem B: Suppose that $u \in W^{j, p}$, with $p<\frac{N}{j}$; then $u \in L\left(p^{*}, p\right)$ with $\frac{1}{p^{*}}=\frac{1}{p}-\frac{j}{N}$.

Note that this result improves Sobolev's theorem, which gives $u \in L^{p^{*}}=L\left(p^{*}, p^{*}\right)$, which is a larger space than $L\left(p^{*}, p\right)$.

The following refinement of Trudinger's result (see H. Brezis and S. Wainger [7], H. Brezis [5]) is of particular importance for our considerations:

Theorem C: Assume $\nabla u \in L(N, q)$ for some $1<q<\infty$. Then $e^{|u|^{\frac{q}{q-1}}} \in L^{1}$.

We make the following

Definition Let $\Omega \subset \mathbb{R}^{N}$ be a bounded domain. Assume that $1<p<\infty, 1<q<\infty$, and set

$$
W_{0}^{1} L(p, q)(\Omega)=\operatorname{cl}\left\{u \in C_{0}^{\infty}(\Omega):\|\nabla u\|_{p, q}<\infty\right\}
$$

On $W_{0}^{1} L(p, q)$ we have the following norm

$$
\|u\|_{1 ; p, q}:=\|\nabla u\|_{p, q}
$$

with which $W_{0}^{1} L(p, q)$ becomes a reflexive Banach space.

\subsection{An exponential "critical hyperbola"}

We consider again the system

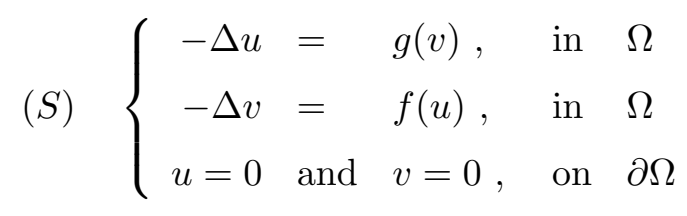

We look for an analogue of the critical hyperbola. One sees by considering the functional on the space $H_{0}^{1} \times H_{0}^{1}$ that the nonlinearities $G(v) \sim e^{|v|^{2}}$ and $F(u) \sim e^{|u|^{2}}$ lie on this "critical curve". We assume that $F(t)$ and $G(t)$ have "exponential polynomial growth", i.e.

$$
F(t) \sim e^{|t|^{p}}, \text { and } G(t) \sim e^{|t|^{q}}, \text { for some } 1<p, q<+\infty .
$$

We prove: 
Theorem 3.1 Let $\Omega \subset \mathbb{R}^{2}$ be a bounded domain. Then we have an "exponential critical curve" given by

$$
(F(s), G(s))=\left(e^{|s|^{p}}, e^{|s|^{q}}\right), \text { with } \frac{1}{p}+\frac{1}{q}=1 .
$$

Proof. We consider the functional

$$
J(u, v)=\int_{\Omega} \nabla u \nabla v d x-\int_{\Omega} F(u)-\int_{\Omega} G(v)
$$

We want to consider the term $\int_{\Omega} \nabla u \nabla v d x$ on a product of Lorentz spaces, i.e. we want to estimate, using the Hölder inequality on Lorentz spaces:

$$
\left|\int_{\Omega} \nabla u \nabla v d x\right| \leq\|\nabla u\|_{L(2, q)}\|\nabla v\|_{L(2, p)}, \frac{1}{q}+\frac{1}{p}=1 .
$$

By Theorem $\mathrm{C}$ we have

$$
e^{|u|^{\frac{q}{q-1}}} \in L^{1} \quad \text { and } \quad e^{|v|^{\frac{p}{p-1}}} \in L^{1}
$$

and thus the maximal growth allowed for $F(s)=\int_{0}^{s} f(t) d t$ and $G(s)=\int_{0}^{s} g(t) d t$ is given by

$$
F(u) \sim e^{|u|^{p}}, p=q^{\prime}=\frac{q}{q-1}, \text { and } G(v) \sim e^{|v|^{q}}, q=p^{\prime}=\frac{p}{p-1}
$$

In other words, Theorem 3.1 says that the maximal growth for system $(S)$ is obtained by the embeddings

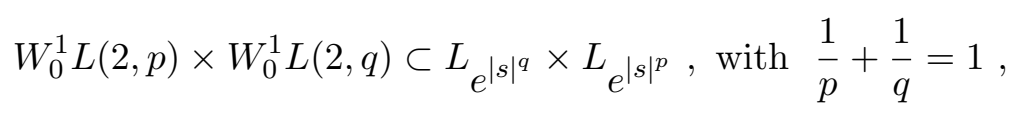

where $L_{\phi}$ denotes the Orlicz space with growth function (N-function) $\phi$.

\subsection{Subcritical growth}

In this section we consider system $(S)$ for nonlinearities $F$ and $G$ with exponential and subcritical growth, i.e.

$$
F(t) \sim e^{|t|^{p}} \text { and } G(t) \sim e^{|t|^{q}}, \text { with } \frac{1}{p}+\frac{1}{q}>1
$$

As mentioned, we consider the functional

$$
J(u, v)=\int_{\Omega} \nabla u \nabla v-\int_{\Omega} F(v)-\int_{\Omega} G(u)
$$

on the space

$$
E=W_{0}^{1} L(2, p) \times W_{0}^{1} L(2, q), \text { with } q=p^{\prime}
$$


We make the following assumptions on $f$ and $g$ (our aim is to give simple assumptions, at the expense of greater generality):

A1) $f$ and $g$ are continuous functions, with $f(s)=o(s)$ and $g(s)=o(s)$ near the origin

A2) There exist constants $\mu>2, \nu>2$ and $s_{0}>0$ such that

$$
0<\mu F(s) \leq s f(s), \text { and } 0<\nu G(s) \leq s g(s), \forall|s| \geq s_{0} .
$$

A3) $F$ and $G$ have at most critical growth, i.e. there exist constants $a_{1}, a_{2}$ and $b_{1}, b_{2}$ such that

$$
F(s) \leq a_{1}+a_{2} e^{|s|^{q^{\prime}}}, G(s) \leq b_{1}+b_{2} e^{|s|^{q}}
$$

A4) Either $F$ or $G$ is subcritical, i.e.

$$
\lim _{|s| \rightarrow \infty} \frac{F(s)}{\Phi(s)}=0 \quad \text { or } \quad \lim _{|s| \rightarrow \infty} \frac{G(s)}{\Psi(s)}=0
$$

where $\Phi(s)=e^{|s|^{q^{\prime}}}$ and $\Psi(s)=e^{|s|^{q}}$.

\section{Example}

$F(s)=e^{|s|^{\alpha}}-1-|s|^{\alpha}, G(s)=e^{|s|^{\beta}}-1-|s|^{\beta}$, with either $1<\alpha<p, 1<\beta \leq q$, or $\alpha=p, 1<\beta<q$.

Theorem 3.2 Under assumptions A1) - A4), system $(S)$ has a nontrivial positive (weak) solution $(u, v) \in E$.

Proof. The proof follows ideas from [15]. The main problems one has to deal with are:

- the functional $J$ given in (12) is strongly indefinite, being unbounded from above and below on infinite dimensional subspaces of $E$

- $E$ is a Banach space, and not (as usual) a Hilbert space

\subsection{Critical growth}

For system $(S)$ with critical growth in the sense of Theorem 3.1 little is known. Only in the particular case when both $F(s)$ and $G(s)$ have the same growth $e^{t^{2}}$, i.e. when one can work on the space $H_{0}^{1}(\Omega) \times H_{0}^{1}(\Omega)$ one has an existence result, see de Figueiredo-doÓ-Ruf [11]:

Theorem 3.3 Assume

(H1) $f$ and $g$ are continuous functions, and that both $f$ and $g$ have critical growth with $\alpha_{0}=4 \pi$;

(H2) $f(s)=o(s)$ and $g(t)=o(t)$ near the origin;

(H3) there exist constants $\theta>2$ and $t_{0}>0$ such that for all $t \geq t_{0}$

$$
0<\theta F(t) \leq t f(t) \text { and } 0<\theta G(t) \leq t g(t) \quad ;
$$


(H4) there exists $M>0$ and $t_{o}>0$ such that, for all $t \geq t_{0}$

$$
0<F(t) \leq M f(t) \text { and } 0<G(t) \leq M g(t) .
$$

(H5) Let $d$ denote the inner radius of $\Omega$, and assume that

$$
\lim _{|t| \rightarrow \infty} \frac{t f(t)}{e^{4 \pi t^{2}}}>\frac{1}{\pi d^{2}} \text { and } \lim _{|t| \rightarrow \infty} \frac{t g(t)}{e^{4 \pi t^{2}}}>\frac{1}{\pi d^{2}}
$$

Then system $(S)$ has a nontrivial weak solution $(u, v) \in E$.

The proof combines the techniques of Theorem 2.2 with the methods of the critical case for systems in dimension $N \geq 3$ (in particular, the problems arising from the indefiniteness of the functional).

We conclude by noting that for nonlinearities with critical growths in the sense of Theorem 3.1 and different from the one considered in Theorem 3.3, many questions remain open. In particular, there are

- no known concentration phenomena

- no existence results and no non-existence results

\section{References}

[1] R. A. Adams, J.J.F. Fournier, Sobolev Spaces, 2nd ed., Academic Press (2003)

[2] Adimurthi, Existence of positive solutions of the semilinear Dirichlet problem with critical growth for the n-Laplacian, Ann. Sc. Norm. Sup. Pisa XVII (1990), 393-413.

[3] A. Ambrosetti, P.H. Rabinowitz, Dual variational methods in critical point theory and applications, J. Functional Analysis 14 (1973), 349-381.

[4] Adimurthi, S.L. Yadava, Multiplicity results for semilinear elliptic equations in a bounded domain of $\mathbb{R}^{2}$ involving criitical exponent, Ann. Sc. Norm. Sup. Pisa XVII (1990), 481-504.

[5] H. Brezis, Laser beams and limiting cases of Sobolev inequalities, Nonlinear Partial Differential Equations and their Applications, Collège de France Seminar, Vol. II, H. Brezis, J.L.Lions ed., Pitman (1982)

[6] H. Brezis and L. Nirenberg, Positive solutions of nonlinear elliptic problems involving critical Sobolev exponent, Comm. Pure App. Math. 36 (1983), 437477.

[7] H. Brezis, S. Wainger, A note on limiting cases of Sobolev embeddings and convolution inequalities, Comm. P.D.E. 5 (1980), 773-789.

[8] Carleson, L., Chang, A., On the existence of an extremal function for an inequality of J. Moser, Bull. Sc. Math. 110 (1986), 113-127.

[9] D. de Figueiredo and P. Felmer, On superquadratic elliptic systems. Trans. Amer. Math. Soc. 343 (1994), 99-116. 
[10] D. G. de Figueiredo, J. M. do Ó and B. Ruf, On an inequality by N. Trudinger and J. Moser and related elliptic equations, Comm. Pure Appl. Math. 55 (2002), 135-152.

[11] D. G. de Figueiredo, J. M. do Ó and B. Ruf, Critical and subcritical elliptic systems in dimension two, Indiana University Math. J. 53 (2004),1037-1054

[12] D. G. de Figueiredo, J. M. do Ó and B. Ruf, An Orlicz space approach to superlinear elliptic systems, J. Functional Analysis, 224 (2005), 471-496

[13] D. G. de Figueiredo and B. Ruf, On the existence and non-existence of solutions for elliptic equations with critical growth in $R^{2}$, Comm. Pure Appl. Math., 48, 1995

$[14]$ D. G. de Figueiredo, O. H. Miyagaki and B. Ruf, Elliptic equations in $\mathbb{R}^{2}$ with nonlinearities in the critical growth range, Calc. Var. 3 (1995), 139-153.

[15] D. G. de Figueiredo, J. M. do Ó and B. Ruf, Critical and subcritical elliptic systems in dimension two, Indiana University Math. J. 53 (2004), 1037-1054.

[16] Flucher, M., Extremal functions for the Trudinger-Moser inequality in 2 dimensions, Comm. Math. Helv. 67 (1992), 471-479.

[17] B. Gidas, W.N. Ni and L. Nirenberg, Symmetry and related properties via maximum principle, Comm. Math. Phys. 68 (1979), 209-243.

[18] J. Hulshof, and R. van der Vorst, Differential systems with strongly indefinite variational structure, J. Funct. Anal. 114 (1993), 32-58.

[19] P.L. Lions, The concentration-compactness principle in the Calculus of Variations, Part I, Revista Iberoamericana 11 (1985), 185-201.

[20] J. Moser, A sharp form of an inequality by N. Trudinger, Ind. Univ. J. 20 (1971), 1077-1092.

[21] S. I. Pohozaev, The Sobolev embedding in the case $p l=n$, Proceedings of the Technical Scientific Conference on Advances of Scientific Research 1964-1965. Mathematics Section, 158-170, Moscov. Ènerget. Inst., Moscow, 1965.

[22] S. I. Pohozaev, On the eigenfunctions of the equation $\Delta u+\lambda f(u)=0$, Dokl. Adad. Nauk SSSR 107, 253-259.

[23] P. H. Rabinowitz, Minimax Methods in Critical Point Theory with Applications to Differential Equations, CBMS Regional Conf. Ser. in Math., 65, AMS, Providence, RI, 1986.

[24] R.S. Strichartz, A note on Trudinger's extension of Sobolev's inequality, Indiana U. Math. J. 21 (1972), 841-842.

[25] G. Talenti, Best constants in Sobolev inequality, Annali Mat. pura appl. 110 (1976), 353372.

[26] N. S. Trudinger, On imbeddings into Orlicz spaces and some applications, J. Math. Mech. 17 (1967), 473-484. 